\title{
Existence of solution of the nonlinear Dirichlet problem for differential-functional equations of elliptic type
}

\author{
by StAnisŁaW BRZYCHCZY (Kraków)
}

Abstract. Consider a nonlinear differential-functional equation

$$
\mathcal{A} u+f(x, u(x), u)=0,
$$

where

$$
\mathcal{A} u:=\sum_{i, j=1}^{m} a_{i j}(x) \frac{\partial^{2} u}{\partial x_{i} \partial x_{j}},
$$

$x=\left(x_{1}, \ldots, x_{m}\right) \in G \subset \mathbb{R}^{m}, G$ is a bounded domain with $C^{2+\alpha}(0<\alpha<1)$ boundary, the operator $\mathcal{A}$ is strongly uniformly elliptic in $G$ and $u$ is a real $L^{p}(\bar{G})$ function.

For the equation (1) we consider the Dirichlet problem with the boundary condition

$$
u(x)=h(x) \quad \text { for } x \in \partial G .
$$

We use Chaplygin's method [5] to prove that problem (1), (2) has at least one regular solution in a suitable class of functions.

Using the method of upper and lower functions, coupled with the monotone iterative technique, H. Amman [3], D. H. Sattinger [13] (see also O. Diekmann and N. M. Temme [6], G. S. Ladde, V. Lakshmikantham, A. S. Vatsala [8], J. Smoller [15]) and I. P. Mysovskikh [11] obtained similar results for nonlinear differential equations of elliptic type.

A special case of (1) is the integro-differential equation

$$
\mathcal{A} u+f\left(x, u(x), \int_{G} u(x) d x\right)=0 .
$$

Interesting results about existence and uniqueness of solutions for this equation were obtained by H. Ugowski [17].

1. Notation, definitions and assumptions. By $C^{l+\alpha}(\bar{G})(l=$ $0,1,2, \ldots ; 0<\alpha \leq 1)$ we denote the space of functions $f \in C^{l}(\bar{G})$ whose

1991 Mathematics Subject Classification: 35J65, 35R10.

Key words and phrases: nonlinear differential-functional equations of elliptic type, monotone iterative technique, Chaplygin's method, Dirichlet problem. 
derivatives of order $l$ are Hölder continuous with finite norm

$$
|f|_{l+\alpha}=\sup _{\substack{x \in G \\|k| \leq l}}\left|D^{k} f(x)\right|+\sup _{\substack{x, y \in G \\|k|=l, x \neq y}} \frac{\left|D^{k} f(x)-D^{k} f(y)\right|}{\|x-y\|^{\alpha}},
$$

where $\|x\|^{2}=\sum_{i=1}^{m} x_{i}^{2}$.

By $H^{m, p}(G)(p \geq 1)$ we denote the Sobolev space (see [1]) defined in the following way: $H^{m, p}(G)$ is the space of all functions $f$ having weak derivatives $D^{\beta} f \in L^{p}(G)$ for all $|\beta| \leq m$ with finite norm

$$
\|f\|_{m, p}=\left(\sum_{|k| \leq m} \int_{G}\left|D^{k} f(x)\right|^{p} d x\right)^{1 / p} .
$$

We assume that the operator $\mathcal{A}$ (see the abstract) is strongly uniformly elliptic, i.e., there is a $\mu>0$ such that

$$
\sum_{i, j=1}^{m} a_{i j}(x) \xi_{i} \xi_{j} \geq \mu\|\xi\|^{2}
$$

for all $x \in G$ and $\xi=\left(\xi_{1}, \ldots, \xi_{m}\right) \in \mathbb{R}^{m}$.

Moreover, we assume that $a_{i j} \in C^{0+\alpha}(\bar{G})$ and $a_{i j}=a_{j i}(i, j=1, \ldots, m)$. The boundary $\partial G$ is assumed to be of class $C^{2+\alpha}$, i.e., a finite union of $C^{2+\alpha}$ surfaces.

We assume that $h \in C^{2+\alpha}(\partial G)$, i.e., there is an $\widetilde{h} \in C^{2+\alpha}(\bar{G})$ such that $\widetilde{h}(x)=h(x)$ for all $x \in \partial G$.

A function $u$ is called regular in $\bar{G}$ if $u \in C^{0}(\bar{G}) \cap C^{2}(G)$.

Functions $u$ and $v$ regular in $\bar{G}$ and satisfying the systems of inequalities

$$
\begin{aligned}
& \begin{cases}\mathcal{A} u+f(x, u(x), u) \geq 0 & \text { for } x \in G, \\
u(x) \leq h(x) & \text { for } x \in \partial G,\end{cases} \\
& \begin{cases}\mathcal{A} v+f(x, v(x), v) \leq 0 & \text { for } x \in G, \\
v(x) \geq h(x) & \text { for } x \in \partial G,\end{cases}
\end{aligned}
$$

are called a lower and an upper functions for problem (1), (2) in $\bar{G}$, respectively.

Assumption A. We assume that there exists at least one pair $u_{0}, v_{0}$ of lower and upper functions for problem (1), (2) in $\bar{G}$ such that

$$
u_{0}(x) \leq v_{0}(x) \text { for } x \in \bar{G} .
$$

Let $u_{0}, v_{0}$ be lower and upper functions for problem (1), (2) in $\bar{G}$. Define

$$
K=\left\{(x, y, s): x \in \bar{G}, y \in\left[m_{0}, M_{0}\right], s \in\left\langle u_{0}, v_{0}\right\rangle\right\},
$$

where

$$
m_{0}=\min _{x \in \bar{G}} u_{0}(x), \quad M_{0}=\max _{x \in \bar{G}} v_{0}(x)
$$


and $\left\langle u_{0}, v_{0}\right\rangle$ is the segment

$$
\left\langle u_{0}, v_{0}\right\rangle:=\left\{s \in L^{p}(G): u_{0}(x) \leq s(x) \leq v_{0}(x) \text { for } x \in G\right\} .
$$

We assume that $f: \mathbb{R}^{m} \times \mathbb{R} \times L^{p} \ni(x, y, s) \mapsto f(x . y, s) \in \mathbb{R}$ satisfies in $K$ the following assumptions:

(a) $f(\cdot, y, s) \in C^{0+\alpha}(\bar{G})$ for $y \in\left[m_{0}, M_{0}\right], s \in\left\langle u_{0}, v_{0}\right\rangle$,

(b) $f(x, \cdot, \cdot)$ is continuous for $x \in \bar{G}$,

(c) the derivative $\partial f / \partial y$ exists and is continuous, and

$$
\left|\frac{\partial f}{\partial y}(x, y, s)\right| \leq c_{0} \quad \text { in } K
$$

where $c_{0}>0$ is a constant,

(d) $f$ is increasing with respect to $s$.

2. Main results. Throughout this paper we assume all assumptions of the first section to hold.

THEOREM 1. The problem (1), (2) has at least one regular solution $u$ such that

$$
u_{0}(x) \leq u(x) \leq v_{0}(x) \quad \text { for } x \in \bar{G} .
$$

Before going into the proof of the theorem we establish some lemmas and make a few remarks.

From assumption (c) it follows that for $k>c_{0}$,

$$
\frac{\partial f}{\partial y}+k>0 \quad \text { in } K \text {. }
$$

Let $\beta$ be a sufficiently regular function defined on $\bar{G}$. Denote by $\mathcal{P}$ the operator $\mathcal{P}: \beta \mapsto \gamma=\mathcal{P} \beta$, where $\gamma$ is the (supposedly unique) solution of the boundary value problem

$$
\begin{cases}(\mathcal{A}-k \mathcal{I}) \gamma=-[f(x, \beta(x), \beta)+k \beta(x)] & \text { in } G, \\ \gamma(x)=h(x) & \text { on } \partial G .\end{cases}
$$

The operator $\mathcal{P}$ is the composition of the nonlinear operator $\mathcal{F}: \beta \mapsto \delta$, where

$$
\mathcal{F} \beta(x):=-[f(x, \beta(x), \beta)+k \beta(x)]=\delta(x)
$$

and the linear operator $\mathcal{G}: \delta \mapsto \gamma$, where $\gamma$ is the (supposedly unique) solution of the linear problem

$$
\begin{cases}(\mathcal{A}-k \mathcal{I}) \gamma=\delta(x) & \text { in } G \\ \gamma(x)=h(x) & \text { on } \partial G\end{cases}
$$

$\mathcal{F}$ is the Nemytskiu operator. It is sometimes also called the superposition operator, composition operator, or substitution operator. More information about it can be found in [4]. 
Lemma 1. (i) $\mathcal{F}$ maps $C^{0+\alpha}(\bar{G})$ into $C^{0+\alpha}(\bar{G})$ and is a bounded and continuous operator between these spaces.

(ii) $\mathcal{P}$ maps $C^{0+\alpha}(\bar{G})$ into $C^{0+\alpha}(\bar{G})$ and is compact.

Proof. Assumption (c) implies that $f$ satisfies the Lipschitz condition with respect to $y$. Therefore arguing as in $[8,7]$ we get (i).

Since the operator $\mathcal{A}$ is strongly uniformly elliptic, $a_{i j} \in C^{0+\alpha}(\bar{G})$, the domain $G$ is bounded, $\partial G \in C^{2+\alpha}, h \in C^{2+\alpha}(\partial G)$ and $\delta \in C^{0+\alpha}(\bar{G})$, by the Schauder theorem [14] (see [9]) problem (8) has a unique solution $\gamma \in C^{2+\alpha}(\bar{G})$ such that

$$
|\gamma|_{2+\alpha} \leq c_{1}\left(|\delta|_{0+\alpha}+|\widetilde{h}|_{2+\alpha}\right)
$$

where $c_{1}>0$ is independent of $\delta$ and $h$.

We define a constant operator $\mathcal{G}_{1}: C^{0+\alpha}(\bar{G}) \rightarrow C^{2+\alpha}(\bar{G})$ by denoting, for every $h \in C^{0+\alpha}(\bar{G})$, by $\mathcal{G}_{1}(h)$ the unique solution of problem (8) with $\delta(x)=0$ in $\bar{G}$.

Similarly, we define a linear operator $\mathcal{G}_{2}: C^{0+\alpha}(\bar{G}) \rightarrow C^{2+\alpha}(\bar{G})$ by denoting, for every $\delta \in C^{0+\alpha}(\bar{G})$, by $\mathcal{G}_{2}(\delta)$ the unique solution of problem (8) with $h(x)=0$ on $\partial G$.

It is easy to see that $\mathcal{G}(\delta)=\mathcal{G}_{1}(h)+\mathcal{G}_{2}(\delta)$. It follows from (9) that $\mathcal{G}_{2}$ is continuous. Consequently, since $\mathcal{G}_{1}$ is constant with respect to $\delta, \mathcal{G}$ is continuous. Thus the operator

$$
\mathcal{G} \circ \mathcal{F}: C^{0+\alpha}(\bar{G}) \rightarrow C^{2+\alpha}(\bar{G})
$$

is bounded and continuous.

Since $\partial G \in C^{2+\alpha}$, the identity operator

$$
\mathcal{I}: C^{2+\alpha}(\bar{G}) \rightarrow C^{0+\alpha}(\bar{G})
$$

is compact (see [19]). Hence the operator

$$
\mathcal{P}=\mathcal{I} \circ \mathcal{G} \circ \mathcal{F}: C^{0+\alpha}(\bar{G}) \rightarrow C^{0+\alpha}(\bar{G})
$$

is compact. This completes the proof of Lemma 1.

LEMMA 2. (i) $\mathcal{F}$ induces a bounded and continuous operator $L^{p}(G) \rightarrow$ $L^{p}(G)$.

(ii) $\mathcal{P}$ induces a compact operator $L^{p}(G) \rightarrow L^{p}(G)$.

Proof. Recall that $G$ is bounded and $f$ satisfies assumptions (a)-(c). Assumption (c) implies that $f$ satisfies the Lipschitz condition with respect to $y$. Therefore arguing as in $[18,7]$ (see also [16]) we conclude that $\mathcal{F}$ maps $L^{p}(G)$ into $L^{p}(G)$. Hence the nonlinear operator $\mathcal{F}$ is bounded and continuous.

If $\delta \in L^{p}(G)$, then using the Agmon-Douglis-Nirenberg theorem [2] (see [9]) and repeating the same arguments as in the proof of Lemma 1, we 
can show that problem (8) has a unique weak solution $\gamma \in H^{2, p}(G)$, which satisfies

$$
\|u\|_{2, p} \leq c_{2}\left(\|\delta\|_{L^{p}}+\|\widetilde{h}\|_{2, p}\right),
$$

where $c_{2}>0$ and $c_{2}$ does not depend on $\delta$ and $h$. Hence

$$
\mathcal{G}: L^{p}(G) \rightarrow H^{2, p}(G) \text {. }
$$

By (10) and using a similar argument to the proof of Lemma 1 one can show that the operator $\mathcal{G}$ is continuous. Thus $\mathcal{G} \circ \mathcal{F}: L^{p}(G) \rightarrow H^{2, p}(G)$ is bounded and continuous. Since the identity operator $\mathcal{I}: H^{2, p}(G) \rightarrow L^{p}(G)$ is compact (see [20]), the composition $\mathcal{P}=\mathcal{I} \circ \mathcal{G} \circ \mathcal{F}: L^{p}(G) \rightarrow L^{p}(G)$ is compact. This completes the proof of Lemma 2 .

LEMmA 3. (i) Let $\beta_{1}$ and $\beta_{2}$ be any regular functions such that $\beta_{1}, \beta_{2}$ $\in K$. Then the operator $\mathcal{P}$ is increasing, i.e., $\beta_{1}(x)<\beta_{2}(x)$ in $G$ implies $\mathcal{P} \beta_{1}(x)<\mathcal{P} \beta_{2}(x)$ in $G$.

(ii) If $\beta$ is an upper (resp. a lower) function for problem (1), (2) in $\bar{G}$, then $\mathcal{P} \beta(x)<\beta(x)$ (resp. $\mathcal{P} \beta(x)>\beta(x))$ in $G$.

Proof. (i) Let $\beta_{1}(x)<\beta_{2}(x)$ in $G$. Setting $\gamma_{1}=\mathcal{P} \beta_{1}$ and $\gamma_{2}=\mathcal{P} \beta_{2}$ from (8) it follows that

$$
\left\{\begin{aligned}
&(\mathcal{A}-k \mathcal{I})\left(\gamma_{2}-\gamma_{1}\right)=-\left[f\left(x, \beta_{2}(x), \beta_{2}\right)-f\left(x, \beta_{1}(x), \beta_{1}\right)\right] \\
&-k\left[\beta_{2}(x)-\beta_{1}(x)\right] \text { in } G \\
& \gamma_{2}(x)-\gamma_{1}(x)=0 \quad \text { on } \partial G .
\end{aligned}\right.
$$

From this, by the monotonicity of $f$ with respect to $s$ we get

$$
\begin{aligned}
(\mathcal{A}-k \mathcal{I}) & \left(\gamma_{2}-\gamma_{1}\right) \\
& \leq-\left[f\left(x, \beta_{2}(x), \beta_{1}\right)-f\left(x, \beta_{1}(x), \beta_{1}\right)\right]-k\left(\beta_{2}(x)-\beta_{1}(x)\right) \\
& =-\left[f_{y}\left(x, \beta_{1}(x)+\theta\left(\beta_{2}(x)-\beta_{1}(x)\right), \beta_{1}\right)+k\right]\left(\beta_{2}(x)-\beta_{1}(x)\right),
\end{aligned}
$$

where $0<\theta<1$. Consequently, by (5) we have

$$
\begin{cases}(\mathcal{A}-k \mathcal{I})\left(\gamma_{2}-\gamma_{1}\right) \leq 0 & \text { in } G \\ \gamma_{2}(x)-\gamma_{1}(x)=0 & \text { on } \partial G .\end{cases}
$$

By the strong maximum principle [12], either $\gamma_{2}(x)-\gamma_{1}(x) \equiv 0$ or $\gamma_{2}(x)-$ $\gamma_{1}(x)>0$ in $G$.

We claim that $\gamma_{2}(x)-\gamma_{1}(x)>0$. Indeed, suppose for a contradiction that $\gamma_{2}(x)-\gamma_{1}(x) \equiv 0$; then by $(11), \beta_{2}(x)-\beta_{1}(x) \equiv 0$ in $G$, contrary to our assumption that $\beta_{1}(x)<\beta_{2}(x)$.

(ii) Putting $\gamma=\mathcal{P} \beta$ and using (6) and (4) we get

$$
\begin{aligned}
(\mathcal{A}-k \mathcal{I})(\gamma-\beta) & =(\mathcal{A}-k \mathcal{I}) \gamma-(\mathcal{A}-k \mathcal{I}) \beta \\
& =-[f(x, \beta(x), \beta)+k \beta(x)]-\mathcal{A} \beta+k \beta(x) \\
& =-[\mathcal{A} \beta+f(x, \beta(x), \beta)] \geq 0 \quad \text { in } G
\end{aligned}
$$


and

$$
\gamma(x)-\beta(x)=h(x)-\beta(x) \leq 0 \quad \text { on } \partial G .
$$

Hence, by the strong maximum principle, either $\gamma(x)-\beta(x) \equiv 0$ or $\gamma(x)-$ $\beta(x)>0$ in $G$. Since $\beta$ is not a solution of (1) (when $\beta$ is a solution of (1) then Theorem 1 holds), the case $\gamma(x)-\beta(x) \equiv 0$ cannot occur. Hence $\gamma(x)<\beta(x)$ in $G$ and the proof of Lemma 3 is complete.

Proof of Theorem 1. Let $\mathcal{P}$ be defined as before. By induction, we define two sequences of functions $\left\{u_{n}\right\}$ and $\left\{v_{n}\right\}$ by setting

$$
\begin{array}{ll}
u_{1}=\mathcal{P} u_{0}, & u_{n}=\mathcal{P} u_{n-1}, \quad n=1,2, \ldots, \\
v_{1}=\mathcal{P} v_{0}, & v_{n}=\mathcal{P} v_{n-1}, \quad n=1,2, \ldots
\end{array}
$$

Now we show that $\left\{u_{n}\right\}$ is increasing (resp. $\left\{v_{n}\right\}$ is decreasing) and converges to a solution of problem (1), (2) in $\bar{G}$. Since $u_{0}$ and $v_{0}$ are regular, by Lemma 1 we see that $u_{n}, v_{n} \in C^{2+\alpha}(\bar{G})$. Since $v_{0}$ is an upper function for problem (1), (2) in $G$, by Lemma 3 , we obtain

$$
v_{1}(x)=\mathcal{P} v_{0}(x)<v_{0}(x) \quad \text { in } G .
$$

Consequently, by monotonicity of $\mathcal{P}$ we get

$$
v_{n}(x)=\mathcal{P} v_{n-1}(x)<v_{n-1}(x) \quad \text { in } G, n=1,2, \ldots
$$

Arguing as above we get $u_{n-1}(x)<u_{n}(x)$ in $\bar{G}, n=1,2, \ldots$ Since the operator $\mathcal{P}$ is monotone, by Assumption A it follows that

$$
u_{1}(x)=\mathcal{P} u_{0}(x) \leq \mathcal{P} v_{0}(x)=v_{1}(x) \quad \text { in } \bar{G}
$$

and consequently $u_{n}(x) \leq v_{n}(x)$ in $\bar{G}, n=1,2, \ldots$ Therefore we get

(13) $u_{0}(x)<u_{1}(x)<\ldots<u_{n}(x)<\ldots<v_{n}(x)<\ldots<v_{1}(x)<v_{0}(x)$ in $\bar{G}$.

By virtue of (13) we can set

$$
\bar{v}(x)=\lim _{n \rightarrow \infty} v_{n}(x) \quad \text { for each } x \in \bar{G}
$$

and we see that $u_{0}(x) \leq \bar{v}(x) \leq v_{0}(x)$ for $x \in \bar{G}$. Analogously we can define

$$
\underline{u}(x)=\lim _{n \rightarrow \infty} u_{n}(x) \text { for each } x \in \bar{G},
$$

which satisfies $u_{0}(x) \leq \underline{u}(x) \leq v_{0}(x)$ for $x \in \bar{G}$.

To complete the proof we must show that $\underline{u}$ and $\bar{v}$ are regular solutions of problem (1), (2) in $\bar{G}$.

If we could prove that the sequences $\left\{u_{n}\right\}$ and $\left\{v_{n}\right\}$ are bounded in $C^{0+\alpha}(\bar{G})$, then since the operator $\mathcal{P}$ is compact and monotone, the sequences $\left\{\mathcal{P} u_{n}\right\}$ and $\left\{\mathcal{P} v_{n}\right\}$ would be convergent in $C^{0+\alpha}(\bar{G})$.

Since it is not possible to prove that for any elliptic operator $\mathcal{A}$ the sequences $\left\{u_{n}\right\}$ and $\left\{v_{n}\right\}$ are bounded in $C^{0+\alpha}(\bar{G})$, we must find another way. 
The inequality (13) implies that $\left\{u_{n}\right\}$ and $\left\{v_{n}\right\}$ are bounded in $L^{p}(G)$. Since $\mathcal{P}$ is increasing and compact in $L^{p}(G)$ (see Lemma 2), the sequences $\left\{\mathcal{P} u_{n}\right\}$ and $\left\{\mathcal{P} v_{n}\right\}$ are converging in $L^{p}(G)$. It is easy to see that

$$
\underline{u}=\lim _{n \rightarrow \infty} \mathcal{P} u_{n}=\lim _{n \rightarrow \infty} \mathcal{P}^{2} u_{n-1}=\mathcal{P} \underline{u} \in L^{p}(G)
$$

and

$$
\bar{v}=\lim _{n \rightarrow \infty} \mathcal{P} v_{n}=\lim _{n \rightarrow \infty} \mathcal{P}^{2} v_{n-1}=\mathcal{P} \bar{v} \in L^{p}(G) .
$$

Since $\underline{u}, \bar{v} \in L^{p}(G)$ and

$$
\begin{aligned}
& \mathcal{G} \circ \mathcal{F} \underline{u}=\underline{u}, \\
& \mathcal{G} \circ \mathcal{F} \bar{v}=\bar{v},
\end{aligned}
$$

by the Agmon-Douglis-Nirenberg theorem we obtain

$$
\underline{u}, \bar{v} \in H^{2, p}(G) .
$$

Now using the well known fact that for $p>m$ the Sobolev space $H^{2, p}(G)$ is continuously imbedded in $C^{0+\alpha}(\bar{G}), 0<\alpha<1$ (see [9]), and by (18) we get

$$
\underline{u}, \bar{v} \in C^{0+\alpha}(\bar{G}) \text {. }
$$

Applying now the Schauder theorem to the equalities (16), (17) and by (19) we get

$$
\underline{u}, \bar{v} \in C^{2+\alpha}(\bar{G}) .
$$

Hence $\underline{u}$ and $\bar{v}$ are regular solutions of problem (1), (2) in $\bar{G}$. Moreover, since the sequences $\left\{u_{n}\right\},\left\{v_{n}\right\}$ are monotone, by (13)-(15) we see that

$$
u_{0}(x) \leq \underline{u}(x) \leq \bar{v}(x) \leq v_{0}(x) \text { for } x \in \bar{G} .
$$

In general $\underline{u}(x) \neq \bar{v}(x)$.

Remark 1 . The solutions $\underline{u}$ and $\bar{v}$ are minimal and maximal solutions of problem (1), (2) in the set $K$, i.e., if $w$ is any solution of problem (1), (2) such that $u_{0}(x) \leq w(x) \leq v_{0}(x)$, then $\underline{u}(x) \leq w(x) \leq \bar{v}(x)$ in $\bar{G}$.

Indeed, if $w$ is such a solution, then $w=\mathcal{P} w$. Hence, by monotonicity of $\mathcal{P}$ we have

$$
w(x)=\mathcal{P} w(x) \leq \mathcal{P} v_{0}(x)=v_{1}(x) \text { in } \bar{G} .
$$

By induction we get $w(x) \leq v_{n}(x)$ in $\bar{G}$, so $w(x) \leq \lim _{n \rightarrow \infty} v_{n}(x)=\bar{v}(x)$ in $\bar{G}$.

Arguing as above we obtain $\underline{u}(x)=\lim _{n \rightarrow \infty} u_{n}(x) \leq w(x)$ in $\bar{G}$.

R e m a r k 2. Uniqueness of solution for a system of differential-functional equations of elliptic type has been studied by M. Malec [10]. He gave some criterion for uniqueness under stronger assumptions.

Acknowledgements. The author is grateful to the referee for valuable comments that greatly improved the original version of this paper. 


\section{References}

[1] R. A. Adams, Sobolev Spaces, Academic Press, New York 1975.

[2] S. Agmon, A. Douglis and L. Nirenberg, Estimates near the boundary for solutions of elliptic partial differential equations satisfying general boundary conditions, Comm. Pure Appl. Math. 12 (1959), 623-727.

[3] H. Amman, On the existence of positive solutions of nonlinear elliptic boundary value problems, Indiana Univ. Math. J. 21 (1971), 125-146.

[4] J. Appell and P. Zabre ̌̌ko, Nonlinear Superposition Operators, Cambridge University Press, Cambridge 1990.

[5] S. Brzychczy, Chaplygin's method for a system of nonlinear parabolic differentialfunctional equations, Differentsial'nye Uravneniya 22 (1986), 705-708 (in Russian).

[6] O. Diekmann and N. M. Temme, Nonlinear Diffusion Problems, MC Syllabus 28, Mathematisch Centrum, Amsterdam 1982.

[7] M. A. Krasnosel'skiı̌, Topological Methods in the Theory of Nonlinear Integral Equations, Pergamon Press, Oxford 1963.

[8] G. S. Ladde, V. Lakshmikantham and A. S. Vatsala, Monotone Iterative Techniques for Nonlinear Differential Equations, Pitman, Boston 1985.

[9] O. A. Ladyzhenskaya and N. N. Ural'ceva, Linear and Quasilinear Elliptic Equations, Academic Press, New York 1968.

[10] M. Malec, Unicité des solutions d'un système non linéaire d'équations elliptiques contenant des fonctionnelles, Boll. Un. Mat. Ital. (6) 2-A (1983), 321-329.

[11] I. P. Mysovskikh, Application of Chaplygin's method to the Dirichlet problem for elliptic equations of a special type, Dokl. Akad. Nauk SSSR 99 (1) (1954), 13-15 (in Russian).

[12] M. H. Protter and H. F. Weinberger, Maximum Principles in Differential Equations, Springer, New York 1984.

[13] D. H. Sattinger, Monotone methods in nonlinear elliptic and parabolic boundary value problems, Indiana Univ. Math. J. 21 (1972), 979-1000.

[14] J. Schauder, Über lineare elliptische Differentialgleichungen zweiter Ordnung, Math. Z. 38 (1934), 257-282.

[15] J. Smoller, Shock Waves and Reaction-Diffusion Equations, Springer, New York 1983.

[16] N. M. Temme (ed.), Nonlinear Analysis, Vol. II, MC Syllabus 26.2, Mathematisch Centrum, Amsterdam 1976

[17] H. Ugowski, On integro-differential equations of parabolic and elliptic type, Ann. Polon. Math. 22 (1970), 255-275.

[18] M. M. Vainberg, Variational Methods for the Study of Nonlinear Operators, Holden-Day, San Francisco 1964.

[19] J. Wloka, Funktionalanalysis und Anwendungen, de Gruyter, Berlin 1971.

[20] -, Grundräume und verallgemeinerte Funktionen, Lecture Notes in Math. 82, Springer, Berlin 1969.

INSTITUTE OF MATHEMATICS

UNIVERSITY OF MINING AND METALLURGY

AL. MICKIEWICZA 30

30-059 KRAKÓW, POLAND

Reçu par la Rédaction le 12.5.1992

Révisé le 8.9.1992 et 20.2.1993 